\title{
ON THE SHARPNESS OF FEWNOMIAL BOUNDS AND THE NUMBER OF COMPONENTS OF FEWNOMIAL HYPERSURFACES
}

\author{
FRÉDÉRIC BIHAN*, J. MAURICE ROJAS ${ }^{\dagger}$, AND FRANK SOTTILE
}

Key words. Fewnomials, connected component

AMS(MOS) subject classifications. 14P99

Abstract. We prove the existence of systems of $n$ polynomial equations in $n$ variables with a total of $n+k+1$ distinct monomial terms possessing $\left\lfloor\frac{n+k}{k}\right\rfloor^{k}$ nondegenerate positive solutions. This shows that the recent upper bound of $\frac{e^{2}+3}{4} 2^{\left(\begin{array}{l}k \\ 2\end{array}\right)} n^{k}$ for the number of nondegenerate positive solutions has the correct order for fixed $k$ and large $n$. We also adapt a method of Perrucci to show that there are fewer than $\frac{e^{2}+3}{4} 2\left(\begin{array}{c}k+1 \\ 2\end{array}\right) 2^{n} n^{k+1}$ connected components in a smooth hypersurface in the positive orthant of $\mathbb{R}^{N}$ defined by a polynomial with $n+k+1$ monomials, where $n$ is the dimension of the affine span of the exponent vectors. Our results hold for polynomials with real exponents.

1. Introduction. Khovanskii's Theorem on Real Fewnomials [5] im-

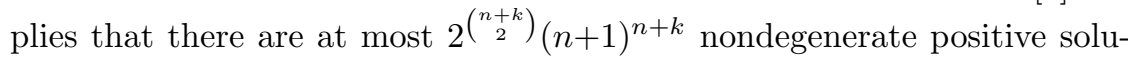
tions to a system of $n$ polynomial equations in $n$ variables which are linear combinations of (the same) $n+k+1$ monomials. This fewnomial bound is also valid in the more general setting of linear combinations of monomials with real-number exponents. The underlying bounds are identical whether one uses integral or real exponents [8], and the arguments of [3] require that we allow real exponents.

While Khovanskii's fewnomial bound was not believed to be sharp, only recently have smaller bounds been found. The first breakthrough was due to Li, Rojas, and Wang [7] who showed that a system of two trinomials in two variables has at most 5 positive solutions - which is smaller than Khovanskii's bound of 5184. Bihan [2] showed that a system of $n$ polynomials in $n$ variables with $n+2$ monomials has at most $n+1$ nondegenerate positive solutions and proved the existence of such a system with $n+1$ positive solutions. Bihan and Sottile [3] generalized this to all $k$, giving the upper bound of $\frac{e^{2}+3}{4} 2_{\left(\begin{array}{l}k \\ 2\end{array}\right)} n^{k}$ for the number of nondegenerate positive solutions, which is significantly smaller than Khovanskii's bound.

*Laboratoire de Mathématiques, Université de Savoie, 73376 Le Bourget-du-Lac Cedex, France; Frederic.Bihan@univ-savoie.fr, ww. lama.univ-savoie.fr/ bihan

${ }^{\dagger}$ Department of Mathematics, Texas A\&M University, College Station, Texas 778433368, USA; rojas@math.tamu.edu, www.math.tamu.edu/ rojas. Rojas supported by NSF CAREER grant DMS-0349309.

$\ddagger$ Department of Mathematics, Texas A\&M University, College Station, Texas 778433368, USA; sottile@math.tamu.edu; www.math.tamu.edu/ sottile. Sottile supported by NSF CAREER grant DMS-0538734. 
1.1. A Lower Bound for Fewnomial Systems. We show that the Bihan-Sottile upper bound [3] is near-optimal for fixed $k$ and large $n$.

THEOREM 1. For any positive integers $n, k$ with $n>k$, there exists a system of $n$ polynomials in $n$ variables involving $n+k+1$ distinct monomials and having $\left\lfloor\frac{n+k}{k}\right\rfloor^{k}$ nondegenerate positive solutions.

We believe that there is room for improvement in the dependence on $k$, both in the upper bound of [3] and in the lower bound of Theorem 1.

Proof. We will construct such a system when $n=k m$, a multiple of $k$, from which we may deduce the general case as follows. Suppose that $n=k m+j$ with $1 \leq j<k$ and we have a system of $m k$ equations in $m k$ variables involving $m k+k+1$ monomials with $(m+1)^{k}$ nondegenerate positive solutions. We add $j$ new variables $x_{1}, \ldots, x_{j}$ and $j$ new equations $x_{1}=1, \ldots, x_{j}=1$. Since the polynomials in the original system may be assumed to have constant terms, this gives a system with $n$ polynomials in $n$ variables having $n+k+1$ monomials and $(m+1)^{k}=\left\lfloor\frac{n+k}{k}\right\rfloor^{k}$ nondegenerate positive solutions. So let us fix positive integers $k, m$ and set $n=k m$.

Bihan [2] showed there exists a system of $m$ polynomials in $m$ variables

$$
f_{1}\left(y_{1}, \ldots, y_{m}\right)=\cdots=f_{m}\left(y_{1}, \ldots, y_{m}\right)=0
$$

having $m+1$ solutions, and where each polynomial has the same $m+2$ monomials, one of which we may take to be a constant.

For each $j=1, \ldots, k$, let $y_{j, 1}, \ldots, y_{j, m}$ be $m$ new variables and consider the system

$$
f_{1}\left(y_{j, 1}, \ldots, y_{j, m}\right)=\cdots=f_{m}\left(y_{j, 1}, \ldots, y_{j, m}\right)=0,
$$

which has $m+1$ positive solutions in $\left(y_{j, 1}, \ldots, y_{j, m}\right)$. As the sets of variables are disjoint, the combined system consisting of all $\mathrm{km}$ polynomials in all $k m$ variables has $(m+1)^{k}$ positive solutions. Each subsystem has $m+2$ monomials, one of which is a constant. Thus the combined system has $1+k(m+1)=k m+k+1=n+k+1$ monomials.

REMARK 1.1. Our proof of Theorem 1 is based on Bihan's nonconstructive proof of the existence of a system of $n$ polynomials in $n$ variables having $n+2$ monomials and $n+1$ nondegenerate positive solutions. While finding such systems explicitly is challenging in general, let us do so for $n \in\{2,3\}$.

The system of $n=2$ equations with 2 variables

$$
x^{2} y-\left(1+4 x^{2}\right)=x y-\left(4+x^{2}\right)=0,
$$

has $4=2+1+1$ monomials and exactly 3 complex solutions, each of which is nondegenerate and lies in the positive quadrant. We give numerical approximations, computed with the computer algebra system SINGULAR $[4,10]$,

$$
(2.618034,4.1459), \quad(1,5), \quad(0.381966,10.854102) .
$$


The system of $n=3$ equations with 3 variables

$$
y z-\left(1 / 8+2 x^{2}\right)=x y-\left(1 / 220+x^{2}\right)=z-\left(1+x^{2}\right)=0,
$$

has $5=3+1+1$ monomials and exactly 4 complex solutions, each of which is nondegenerate and lies in the positive octant,

$$
\begin{array}{rc}
(0.076645,0.1359,1.00587), & (0.084513,0.13829,1.00714), \\
(0.54046,0.54887,1.2921), & (1.29838,1.30188,2.6858) .
\end{array}
$$

1.2. An Upper Bound for Fewnomial Hypersurfaces. Khovanskii also considered smooth hypersurfaces in the positive orthant $\mathbb{R}_{>}^{n}$ defined by polynomials with $n+k+1$ monomials. He showed [6, Sec. 3.14, Cor. 4] that the total Betti number of such a fewnomial hypersurface is at most

$$
\left(2 n^{2}-n+1\right)^{n+k}(2 n)^{n-1} 2^{\left(\begin{array}{c}
n+k \\
2
\end{array}\right)} .
$$

Li, Rojas, and Wang [7] bounded the number of connected components of such a hypersurface by $n(n+1)^{n+k+1} 2^{n-1} 2_{2}^{\left(\begin{array}{c}n+k+1 \\ 2\end{array}\right)}$. Perrucci [9] lowered

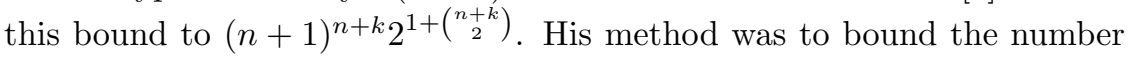
of compact components and then use an argument based on the faces of the $n$-dimensional cube to bound the number of all components. We improve Perrucci's method, using the $n$-simplex and the bounds of Bihan and Sottile [3] to obtain a new, lower bound.

THEOREM 2. A smooth hypersurface in $\mathbb{R}_{>}^{N}$ defined by a polynomial with $n+k+1$ monomials whose exponent vectors have $n$-dimensional affine span has fewer than

$$
\frac{e^{2}+3}{4} \cdot 2^{\left(\begin{array}{c}
k+1 \\
2
\end{array}\right)} 2^{n} n^{k+1}
$$

connected components.

Both our method and that of Perucci estimate the number of compact components in the intersection of the hypersurface with linear spaces which are chosen to preserve the sparse structure so that the fewnomial bound still applies. For this same reason, this same method was used by Benedetti, Loeser, and Risler [1] to bound the number of components of a real algebraic set in terms of the degrees of its defining polynomials.

Observe that if the exponent vectors of the monomials in a polynomial $f$ in $N$ variables have $n$-dimensional affine span, then there is a monomial change of variables on $\mathbb{R}_{>}^{N}$ so that $f$ becomes a polynomial in the first $n$ variables, and thus our hypersurface becomes a cylinder

$$
\mathbb{R}_{<}^{N-n} \times\left\{x \in \mathbb{R}_{>}^{n} \mid f(x)=0\right\},
$$


which shows that it suffices to prove Theorem 2 when $n=N$. That is, for smooth hypersurfacs in $\mathbb{R}^{n}$ defined by polynomials with $n+k+1$ monomial terms whose exponent vectors affinely span $\mathbb{R}^{n}$.

Let $\kappa(n, k)$ be the maximum number of compact connected components of such a smooth hypersurface and let $\tau(n, k)$ be the maximal number of connected components of such a hypersurface. We deduce Theorem 2 from the following combinatorial lemma.

Lemma 3. $\tau(n, k) \leq \sum_{i=0}^{n-1}\left(\begin{array}{c}n+1 \\ i\end{array}\right) \kappa(n-i, k+1)$.

Proof of Theorem 2. Bihan and Sottile [3] proved that

$$
\kappa(n, k) \leq \frac{e^{2}+3}{8} 2^{\left(\begin{array}{l}
k \\
2
\end{array}\right)} n^{k} .
$$

Substituting this into Lemma 3 bounds $\tau(n, k)$ by

$$
\begin{aligned}
& \frac{e^{2}+3}{8} 2^{\left(\begin{array}{c}
k+1 \\
2
\end{array}\right)} \sum_{i=0}^{n-1}\left(\begin{array}{c}
n+1 \\
i
\end{array}\right)(n-i)^{k+1} \\
& \quad<\frac{e^{2}+3}{8} 2^{\left(\begin{array}{c}
k+1 \\
2
\end{array}\right)} n^{k+1} \sum_{i=0}^{n+1}\left(\begin{array}{c}
n+1 \\
i
\end{array}\right)=\frac{e^{2}+3}{8} 2^{\left(\begin{array}{c}
k+1 \\
2
\end{array}\right)} n^{k+1} 2^{n+1} .
\end{aligned}
$$

Proof of Lemma 3. Let $f$ be a polynomial in the variables $x_{1}, \ldots, x_{n}$ which has $n+k+1$ distinct monomials whose exponent vectors affinely span $\mathbb{R}^{n}$ and suppose that $f(x)=0$ defines a smooth hypersurface $X$ in $\mathbb{R}_{>}^{n}$. We may apply a monomial change of coordinates to $\mathbb{R}_{>}^{n}$ and assume that $1, x_{1}, x_{2}, \ldots, x_{n}$ are among the monomials appearing in $f$.

Suppose that $\varepsilon:=\left(\varepsilon_{0}, \varepsilon_{1}, \ldots, \varepsilon_{n}\right) \in \mathbb{R}_{>}^{1+n}$ with $\varepsilon_{0} \varepsilon_{1} \cdots \varepsilon_{n} \neq 1$. Define hypersurfaces $H_{0}, H_{1}, \ldots, H_{n}$ of $\mathbb{R}_{>}^{n}$ by

$$
\begin{aligned}
H_{0} & :=\left\{x \in \mathbb{R}_{>}^{n} \mid x_{1} \cdots x_{n} \varepsilon_{0}=1\right\} \quad \text { and } \\
H_{i} & :=\left\{x \mid x_{i}=\varepsilon_{i}\right\} \quad \text { for } i=1, \ldots, n .
\end{aligned}
$$

The transformation Log: $\mathbb{R}_{>}^{n} \rightarrow \mathbb{R}^{n}$ defined on each coordinate by $x_{i} \mapsto$ $\log \left(x_{i}\right)$ sends the hypersurfaces $H_{0}, H_{1}, \ldots, H_{n}$ to hyperplanes in general position. That is, if $S \subset\{0,1, \ldots, n\}$ and we define $H_{S}:=\cap_{i \in S} H_{i}$, then $\log \left(H_{S}\right)$ is an affine linear space of dimension $n-|S|$. Moreover, the complement of the union of hypersurfaces $H_{i}$ has $2^{n+1}-1$ connected components, exactly one of which is bounded.

If we restrict $f$ to some $H_{S}$, we will obtain a new polynomial $f_{S}$ in $n-|S|$ variables with at most $1+(n-|S|)+(k+1)$ monomials. Indeed, if $i \in S$ with $i \neq 0$, then the equation $x_{i}=\varepsilon_{i}$ allows us to eliminate both the variable and the monomial $x_{i}$ from $f$. If however $0 \in S$, then we pick an index $j \notin S$ and use $x_{1} \cdots x_{n} \varepsilon_{0}=1$ to eliminate the variable $x_{j}$, which will not necessarily eliminate a monomial from $f$. For almost all $\varepsilon$, the polynomial $f_{S}$ defines a smooth hypersurface $X_{S}$ of $H_{S}$. 
We may choose $\varepsilon$ small enough so that every compact connected component of $X$ lies in the bounded region of the complement of the hypersurfaces $H_{i}$, and every noncompact connected component of $X$ meets some hypersurface $H_{i}$. Shrinking $\varepsilon$ if necessary, we can ensure that every bounded component of $X_{S}$ lies in the bounded region of the complement of $H_{j} \cap H_{S}$ for $j \notin S$, and every unbounded component meets some $H_{j} \cap H_{S}$ for $j \notin S$.

Given a connected component $C$ of $X$, the subsets $S \subset\{0,1, \ldots, n\}$ such that $C$ meets $H_{S}$ form a simplicial complex. If $S$ represents a maximal simplex in this complex, then $C \cap H_{S}$ is a union of compact components of $X_{S}$, and $|S|<n$ as $H_{S}$ is not a point. Thus the number of connected components of $X$ is bounded by the sum of the numbers of compact components of $X_{S}$ for all $S \subset\{0,1, \ldots, n\}$ with $n>|S|$. Since each $f_{S}$ has at most $1+(n-|S|)+(k+1)$ monomials, this sum is bounded by the sum in the statement of the lemma.

REMARK 1.2. If $f$ contains a monomial $x^{a}:=x_{1}^{a_{1}} x_{2}^{a_{2}} \cdots x_{n}^{a_{n}}$ with no $a_{i}=0$, then we can alter the proof of Lemma 3 to obtain a bound of

$$
\frac{e^{2}+3}{4} \cdot 2^{\left(\begin{array}{c}
k \\
2
\end{array}\right)} 2^{n} n^{k}
$$

connected components for the hypersurface defined by $f$.

The basic idea is that if we redefine $H_{0}$ to be

$$
H_{0}:=\left\{x \in \mathbb{R}_{>}^{n} \mid x^{a} \varepsilon_{0}=1\right\} .
$$

then the polynomials $f_{S}$ on $H_{S}$ have only $1+(n-|S|)+k$ monomials, and so we estimate the number of compact components of $X_{S}$ by $\kappa(n-i, k)$ instead of $\kappa(n-i, k+1)$.

Acknowledgment. We thank Alicia Dickenstein and Daniel Perucci whose comments inspired us to find the correct statement and proof of Theorem 2.

\section{REFERENCES}

[1] R. Benedetti, F. Loeser, And J.-J. Risler, Bounding the number of connected components of a real algebraic set, Discrete Comput. Geom., 6 (1991), pp. 191209.

[2] F. Bihan, Polynomial systems supported on circuits and dessins d'enfants, 2005. Journal of the London Mathematical Society, to appear.

[3] F. Bihan AND F. SotTile, New fewnomial upper bounds from Gale dual polynomial systems, 2006. Moscow Mathematical Journal, to appear. math. AG/0609544.

[4] G.-M. Greuel, G. Pfister, and H. Schönemann, Singular 3.0, A Computer Algebra System for Polynomial Computations, Centre for Computer Algebra, University of Kaiserslautern, 2005. http://www.singular.uni-kl.de.

[5] A. Khovanskit, A class of systems of transcendental equations, Dokl. Akad. Nauk. SSSR, 255 (1980), pp. 804-807.

[6] Fewnomials, Trans. of Math. Monographs, 88, AMS, 1991. 
[7] T.-Y. LI, J. M. RoJAs, AND X. WANG, Counting real connected components of trinomial curve intersections and m-nomial hypersurfaces, Discrete Comput. Geom., 30 (2003), pp. 379-414.

[8] D. Napoletani, A power function approach to Kouchnirenko's conjecture, in Symbolic computation: solving equations in algebra, geometry, and engineering (South Hadley, MA, 2000), vol. 286 of Contemp. Math., Amer. Math. Soc., Providence, RI, 2001, pp. 99-106.

[9] D. Perrucci, Some bounds for the number of components of real zero sets of sparse polynomials, Discrete Comput. Geom., 34 (2005), pp. 475-495.

[10] W. Pohl and M. Wenk, solve.lib, 2006. A Singular 3.0 library for Complex Solving of Polynomial Systems. 